\section{Disgeusia como principal manifestación oral en pacientes con COVID-19 leve en el Instituto Nacional de Enfermedades Respiratorias}

\author{
Dysgeusia as the main oral manifestation \\ in patients with mild COVID-19 at the \\ National Institute of Respiratory Diseases
}

\begin{abstract}
Resumen
Objetivo. Reportar las manifestaciones orales más comunes asociadas a COVID-19 así como si estas persisten en un período de 3 meses. Métodos. Se realizó un estudio longitudinal, descriptivo, evaluando a 149 individuos positivos por reacción en cadena de la polimerasa (PCR) para SARS-CoV-2 a los cuales se les realizó un estudio inicial y un control 3 meses después. Resultados. El 65\% de los participantes reportó alguna manifestación en cavidad bucal y el $24 \%$ alguna secuela. Dentro de estos síntomas y secuelas bucales observados, la de mayor frecuencia fue la disgeusia la cual se encontró en $51 \%$ de los pacientes seguida por xerostomía $27 \%$, ganglios o glándulas inflamadas (17\%). Dichas manifestaciones disminuyeron de forma importante en el seguimiento, sin embargo, prevalecieron en algunos pacientes. Conclusiones. La disgeusia es la manifestación oral de mayor frecuencia en este estudio y se presentó entre las cinco manifestaciones generales más comunes.
\end{abstract}

Palabras clave: COVID-19; Manifestaciones bucales; Disgeusia (fuente: DeCS BIREME).

\begin{abstract}
Objective. To report the oral manifestations associated with COVID-19 as well as whether these persist over a period of 3 months. Methods. A longitudinal, descriptive study was carried out evaluating 149 individuals positive for SARS-CoV-2 by polymerase chain reaction (PCR), who underwent an initial examination and a control 3 months later. Results. A total of $65 \%$ of the participants reported some manifestation in the oral cavity and $24 \%$ some sequelae. Within these observed symptoms and oral sequelae, the one with the highest incidence was dysgeusia, which was found in $51 \%$ of patients followed by xerostomia (27\%), lymph nodes or swollen glands $(17 \%)$. These manifestations decreased significantly during the follow-up, however they prevailed in some patients. Conclusions. Dysgeusia is the oral manifestation with the highest incidence in this study and was among the 5 most common general manifestations.
\end{abstract}

Keywords: COVID-19; Oral manifestations; Dysgeusia (source: MeSH NLM).

\section{Artículo Original}

Carlos Alberto Carrasco Rueda 1,a, llan Vinitzky Brener 1,a, Ana Cristina Román Aragón ${ }^{1, b}$, José Antonio Vélez Peralta ${ }^{1, c}$, Valeria Morales Lundes ${ }^{1, c}$, Rosario Fernández Plata ${ }^{2, \mathrm{~d}}$, Anjarath Higuera Iglesias ${ }^{2, e}$

1 Instituto Nacional de Enfermedades Respiratorias "Ismael Cosió Villegas", Servicio de Estomatología, Ciudad de México, México.

${ }^{2}$ Instituto Nacional de Enfermedades Respiratorias "Ismael Cosió Villegas", Departamento de Epidemiología y

Estadística, Ciudad de México, México.

a Especialista en Cirugía oral y Maxilofacial.

${ }^{b}$ Cirujano Dentista.

c Pasante de Servicio Social Cirujano Dentista.

${ }^{-}$Maestría en Ciencias Médicas.

e Médico Cirujano Especialista.

\section{Correspondencia:}

Carlos Alberto Carrasco Rueda: drcarrascor@hotmail.com Calzada de Tlalpan 4502, Col. Sección XVI, Delegación Tlalpan 14080 México, CDMX. Teléfono: 5554871700 Ext. 5163.

ORCID: 0000-0003-4641-4682

\section{Coautores:}

Ilan Vinitzky Brener: ilanvinitzky@hotmail.com ORCID: 0000-0001-8378-1444

Ana Cristina Román Aragón: dracris_ra@hotmail.com ORCID: 0000-0001-9235-8314

José Antonio Vélez Peralta: antonio.vp@live.com ORCID: 0000-0002-5008-4658

Valeria Morales Lundes: valmorlund@hotmail.com ORCID: 0000-0002-3871-758X

Rosario Fernández Plata: rosferpla@gmail.com

ORCID: 0000-0002-2971-0845

Anjarath Higuera Iglesias: higuera.iglesias.anjarath@ gmail.com

ORCID: 0000-0001-7034-9361

Editor: Donald Ramos-Perfecto

Universidad Nacional Mayor de San Marcos, Perú.

Conflicto de intereses: los autores declaran no tener conflictos de interés.

Fuente de financiamiento: autofinanciado.

\section{Recibido: 02/08/21}

Aceptado: 05/11/21

Publicado: 21/01/22 


\section{Introducción}

Las manifestaciones clínicas de la COVID-19 son sistémicas y diversas, y van desde un estado asintomático, el síndrome de dificultad respiratoria aguda, disfunción multiorgánica o incluso la muerte. Asociadas a la enfermedad también se han descrito manifestaciones orales como: alteraciones del gusto o disgeusia, xerostomía, lesiones orales, gingivitis descamativa, petequias y algunas infecciones oportunistas como la candidiasis ${ }^{1,2}$. Sin embargo, se desconoce si estos signos y síntomas son manifestaciones primarias o bien son secundarias al tratamiento médico establecido.

Actualmente se conoce como es el mecanismo de infección y entrada del virus a las células del organismo, en un estudio realizado por Zou y cols. ${ }^{3}$, indican que la enzima convertidora de angiotensina II (ECA2) es el receptor celular de SARS-CoV-2. Por lo tanto, todas las células que expresan ECA2 pueden actuar como células diana y ser susceptibles a la infección por SARS$\mathrm{CoV}-2^{4}$. Tales como células del pulmón, el corazón, riñón, vejiga, endotelio vascular, lengua, glándulas salivales, entre otros ${ }^{5,6}$, lo que podría explicar la transmisión de la infección entre casos asintomáticos, al estar contenido el SARS-CoV-2 en los receptores ECA2 de los acinos glandulares antes de la progresión hacia las vías respiratorias ${ }^{5}$.

El primer reporte de anosmia y disgeusia fue en febrero del 2020 por Mao et al. al estudiar 214 pacientes y reportando que el $36,4 \%$ de ellos describieron estas alteraciones neurológicas ${ }^{6}$. Dos meses después, se publicó un estudio multicéntrico con 417 pacientes, donde el $88,8 \%$ informaron trastornos en la percepción de los sabores salado, dulce, amargo y ácido ${ }^{7,8}$. Dos Santos et al., realizaron una Living Systematic Review (LSR), incluyeron un total de 40 estudios y 10228 pacientes en 19 países, reportaron trastornos del gusto en el $45 \%$ de los pacientes clasificados como disgeusia (38\%), hipogeusia $(35 \%)$ y ageusia (24\%), la conclusión fue que los trastornos del gusto pueden ser un síntoma significativo y específico de los casos de COVID-19 leve / moderado con predilección al sexo femenino ${ }^{1}$.

Las lesiones de mucosa oral se han descrito en lengua, paladar, labios, encía y mucosa bucal. Estas lesiones han sido encontradas en pacientes hospitalizados y sometidos a tratamientos farmacológicos, teniendo su aparición entre los días 7 y 24, después del inicio de los síntomas ${ }^{1}$, por lo que se piensa que su etiología puede ser la terapia farmacológica establecida y/ o por su condición sistémica.

Otras alteraciones orales descritas asociadas a SARS$\mathrm{CoV}-2$ son las lesiones versículo-bulbosas '; Dantas y cols., realizaron biopsias de lesiones presentes en los labios y encontraron un infiltrado linfocitario moderado y trombosis microvascular; en la inmunohistoquímica identificaron la proteína spike en células inflamatorias endoteliales, así como en los queratinocitos y en las células acinares y ductales de glándulas salivales menores ${ }^{10}$.
El objetivo del presente estudio es reportar las principales manifestaciones orales de COVID-19 así como si estas persisten en un período de 3 meses, determinar si las lesiones orales preceden a las manifestaciones sistémicas de la enfermedad, conocer si las manifestaciones orales junto con la pérdida del gusto y del olfato son indicadores tempranos de COVID-19, ayudando a un diagnóstico precoz para disminuir la propagación de la enfermedad.

\section{Métodos}

Se realizó un estudio analítico longitudinal para evaluar manifestaciones orales durante el período inicial de la enfermedad COVID-19 y 3 meses posterior a su diagnóstico. Para ello, se llevaron a cabo estudios epidemiológicos en un periodo comprendido entre junio y diciembre del 2020 a 1200 trabajadores del Instituto Nacional de Enfermedades Respiratorias los cuales acudieron por presentar síntomas asociados a COVID-19o bien por haber tenido contacto con pacientes positivos, a los cuales se realizó prueba de reacción en cadena de la polimerasa (PCR) para detección de SARS-CoV-2. De los 1200 pacientes estudiados 220 tuvieron un resultado positivo para SARS-CoV-2 y se les invitó a participar en el seguimiento. Se incluyeron en el estudio 149 trabajadores positivos para SARS CoV-2, a los que se les realizó el estudio inicial y un seguimiento a los 3 meses. A los participantes se les explicó las características del estudio, así como los objetivos del mismo. Aquellos que no aceptaron participar fueron excluidos del seguimiento y por lo tanto de la muestra total (Figura 1).

La primera entrevista se realizó por video llamada y el estudio de seguimiento fue por vía telefónica, debido a lo anterior la aceptación de participación en el estudio se realizó de forma verbal previa lectura del consentimiento informado.

Tanto en el estudio inicial como en el de seguimiento se interrogó sobre:

Datos generales (edad, sexo, lugar de residencia, ocupación)

Datos clínicos generales (fecha de inicio de síntomas, síntomas que presenta, comorbilidades, peso, talla, medicamentos utilizados)

\section{Manifestaciones orales:}

- Disgeusia: definida com una capacidad reducida o distorsionada para percibir sabores y se englobaron en esta variable la ageusia, hipogeusia, disgeusia y fantogeusia.

- Xerostomía: sensación de boca seca.

- Lesiones en boca: cualquier lesión en cavidad bucal referida por el paciente incluyendo úlceras, vesículas, aftas, nódulos, máculas.

- Inflamación de ganglios o glándulas en cara o cuello: sensación de aumento de volumen en el cuello percibida por el paciente. 
El estudio fue aprobado por el comité de ética del Instituto Nacional de Enfermedades Respiratorias el 29 de abril de 2020 con la asignación de código: E05-20.

Análisis estadístico. Se evaluó la normalidad de la edad con la prueba de Shapiro Wilk. Para comparar la media de la edad entre sexo, se utilizó la prueba t de Student; en cuanto a la comparación de proporciones de los síntomas entre COVID-19 versus post COVID-19, se utilizó la prueba de McNemar para datos pareados. Finalmente, para comparar los síntomas entre sexo, tanto en la medición COVID-19 como en la post COVID-19, se realizó la prueba chi cuadrado de Pearson y la prueba exacta de Fisher para las frecuencias menores a 5. Se utilizó el software estadístico Stata v17.

\section{Resultados}

De los 149 trabajadores que aceptaron participar en el estudio, el 37\% fueron hombres y la edad media de los

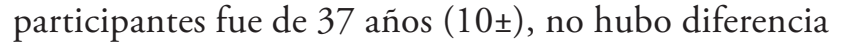

significativa de la edad por sexo (Mujeres $36(10 \pm)$,

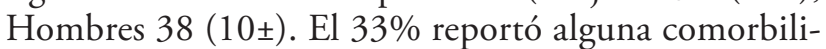
dad siendo las más frecuentes: sobrepeso $(23 \%)$, rinitis $(7,3 \%)$ e hipertensión (6\%), el 67\% de los trabajadores fueron del área de enfermería, $11 \%$ médicos, $18 \%$ administrativos y el resto de diversas áreas del hospital. De los 149 participantes, el $84 \%$ de los trabajadores manifestaron algún síntoma durante su enfermedad COVID-19 y el 75\% reportó una o más secuelas en su entrevista de seguimiento. Se registraron un total de 40 manifestaciones (signos o síntomas) de las cuales las más reportadas fueron fatiga, cefalea, anosmia, mialgias y disgeusia (Tabla 1).

El 65\% (98) de los participantes reportó alguna manifestación en cavidad oral y el 24\% (36) alguna secuela de estas manifestaciones (Figura 2). Dentro de las manifestaciones orales, la de mayor frecuencia fue la disgeusia la cual se encontró en $51 \%$ de los pacientes seguida por xerostomía $27 \%$ y ganglios o glándulas inflamadas

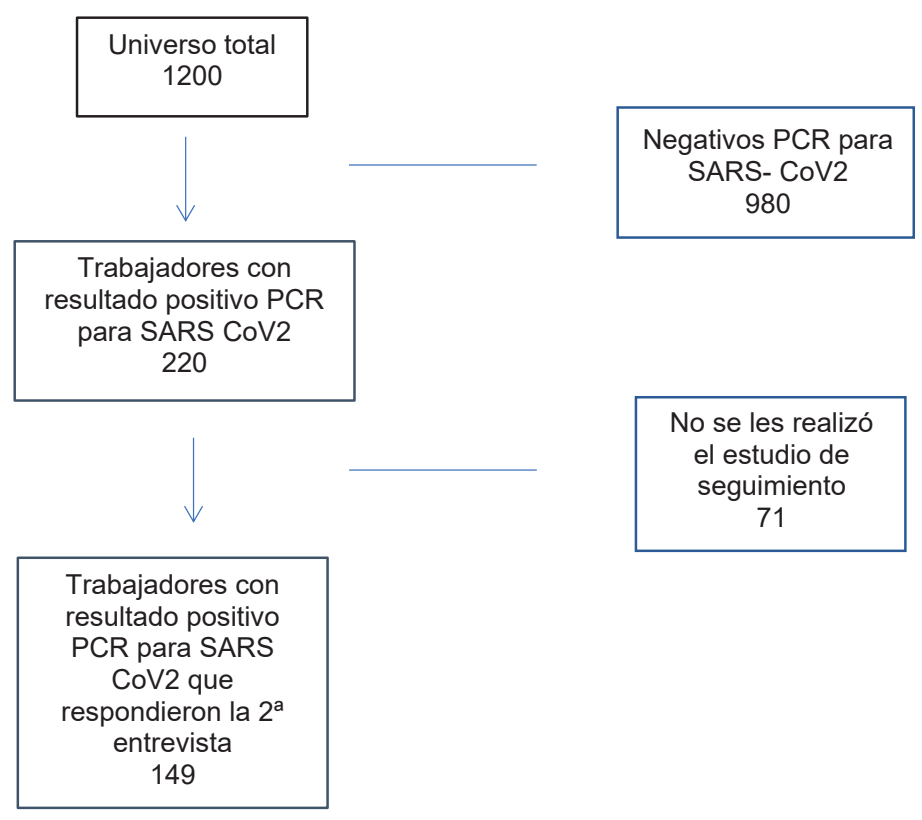

Figura 1. Muestra obtenida para el estudio

Tabla 1. Manifestaciones y secuelas más comunes

\begin{tabular}{lcc}
\hline Manifestación & COVID-19 & Post COVID-19 \\
\hline Fatiga o Debilidad & $95(63 \%)$ & $49(33 \%)$ \\
Cefalea & $89(59 \%)$ & $32(21 \%)$ \\
Anosmia & $82(55 \%)$ & $17(11 \%)$ \\
Mialgias & $80(53 \%)$ & $20(13 \%)$ \\
Disgeusia & $76(51 \%)$ & $14(9.3 \%)$ \\
Artralgias & $63(42 \%)$ & $22(15 \%)$ \\
Escalofríos & $63(42 \%)$ & $15(10 \%)$ \\
Disnea & $56(37 \%)$ & $31(21 \%)$ \\
Tos & $56(37 \%)$ & $9(6 \%)$ \\
Odinofagia & $56(37 \%)$ & $8(5,3 \%)$ \\
\hline
\end{tabular}


17\%. Dichas manifestaciones disminuyeron de forma importante en el cuestionario de seguimiento sin embargo la disgeusia prevaleció en 14 de los participantes que la reportaron en el periodo inicial y la xerostomía en 10 . El 36\% de los participantes reportaron presentar más de una manifestación en boca. El 93\% de los pacientes que reportaron disgeusia también reportó anosmia (Tabla 2).

Analizando la diferencia por sexo, únicamente la disgeusia presentó una diferencia significativa $(\mathrm{p}=0,006)$, siendo más común en las mujeres a diferencia del resto de manifestaciones bucales que no presentaron diferencias (Tabla 3). Dos pacientes presentaron anosmia y disgeusia como únicos síntomas de su enfermedad.

\section{Discusión}

Un $65 \%$ de los pacientes presentaron algún tipo de manifestación oral, las más frecuentes incluyen disgeusia, xerostomía, e inflamación de ganglios y/o glándulas salivales. Cabe mencionar, que los pacientes estudiados no presentaron una forma grave de la enfermedad en la que se requiriera intubación de los pacientes y/o un tratamiento invasivo. Dentro de las manifestaciones orales la alteración del gusto fue la de mayor frecuencia en los casos reportados y concuerda con los resultados de Dos Santos, et al. ${ }^{1}$, en el LSR con un $45 \%$ comparado con nuestro reporte de $51 \%$, en donde también reportan un aparente incremento de la sensibilidad en pacientes de América comparados con los Asiáticos que presentan una menor frecuencia de la manifestación, sugiriendo diferente susceptibilidad o una expresión distinta del receptor ECA2 comparado con los europeos o asiáticos ${ }^{1}$.

Se ha descrito la hipótesis en que la mayoría de las disfunciones gustativas se asocian a alteraciones del olfato ${ }^{11}$, en el estudio realizado el $93 \%$ de los pacientes presentaron esta asociación, queda como interrogante si es una interpretación de los pacientes que al presentar anosmia sea un sinónimo de disgeusia. En cuanto al sexo, se encontró una mayor frecuencia de disgeusia en las mujeres y se ha atribuido a la modulación hormonal y a la respuesta inmune innata a las infecciones virales que aparenta ser exacerbada en las mujeres y podría contribuir a una mayor disfunción quimiosensorial ${ }^{12}$. Los resultados nos indican que la disgeusia es síntoma significativo y/o inicial de COVID-19 en pacientes con el desarrollo de enfermedad leve a moderada; aunque es un reporte breve en cuanto a la muestra y seguimiento

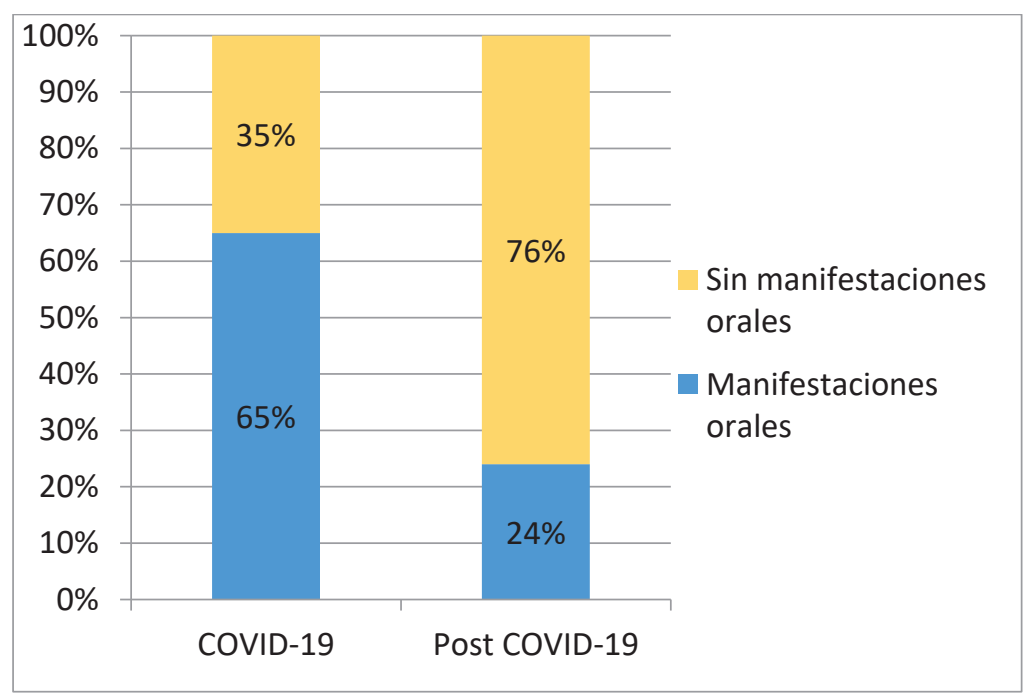

Figura 2. Comparación de manifestaciones orales reportadas durante COVID-19 versus post COVID-19.

Tabla 2. Manifestaciones orales reportadas por trabajadores de la salud, durante la infección a SARS-CoV-2 y posterior a su recuperación aproximadamente 3 meses de salir negativos

\begin{tabular}{llll}
\hline Variable & COVID-19 & Post COVID-19 & \\
\hline & $\mathrm{n}=149$ & & $\mathrm{n}=149$ \\
\hline Disgueusia & $\mathbf{n}(\mathbf{\%})$ & $\mathbf{n}(\mathbf{\%})$ & Valor $^{*}$ \\
Xerostomía & $76(51)$ & $14(9,4)$ & $<0,001^{* *}$ \\
Lesiones en boca & $40(27)$ & $10(6,7)$ & $<0,001^{* *}$ \\
Ganglios o glándulas inflamados & $10(6,7)$ & $5(3,4)$ & 0,132 \\
\hline
\end{tabular}

* Cada paciente puede presentar más de una manifestación 
Tabla 3. Comparación de síntomas y secuelas bucales por sexo durante COVID-1 versus post COVID-19

\begin{tabular}{lllllll}
\hline \multirow{2}{*}{ Variable } & $\begin{array}{l}\text { Mujeres } \\
\text { COVID-19 }\end{array}$ & $\begin{array}{l}\text { Mujeres } \\
\text { Post COVID-19 }\end{array}$ & & $\begin{array}{l}\text { Hombres } \\
\text { COVID-19 }\end{array}$ & $\begin{array}{l}\text { Hombres Post } \\
\text { COVID-19 }\end{array}$ \\
\cline { 2 - 7 } & $\mathbf{n}=\mathbf{9 4}$ & & Valor $\mathbf{p}^{*}$ & $\mathbf{n}(\%)$ & $\mathbf{n}(\%)$ & Valor $^{*}$ \\
\cline { 2 - 7 } & $\mathbf{n}(\%)$ & $\mathbf{n}(\%)$ & $<0,001^{* *}$ & $20(36) \dagger$ & $4(7,3)$ & $<0,001^{* *}$ \\
Disgeusia & $56(59) \dagger$ & $10(11)$ & $<0,001^{* *}$ & $12(22)$ & $3(5,5)$ & $0,003^{* *}$ \\
Xerostomía & $28(30)$ & $7(7,4)$ & 0,103 & $4(7,3)$ & $3(5,5)$ & 0,655 \\
Gesiones en boca & $6(6,4)$ & $2(2,1)$ & $<0,001^{* *}$ & $6(11)$ & $1(1,8)$ & 0,059 \\
\hline
\end{tabular}

* Un solo paciente puede presentar más de una manifestación

* Prueba McNemar para datos pareados

** Cambio estadísticamente significativo con $\mathrm{p}<0,05$

+ Prueba chi2 de Pearson, entre sexo $(p<0,05)$

podrían ser considerados como indicativos de infección temprana en pacientes con COVID-19 leve a moderado y ayudaría a orientar al que lo padece al autoaislamiento y a la indicación de la detección oportuna de la enfermedad mediante pruebas específicas. La disgeusia se encontró dentro de las cinco principales manifestaciones de los pacientes estudiados. Aunque esta manifestación disminuye de forma considerable en su seguimiento esta prevaleció en algunos casos. Se entiende que los pacientes positivos a SARS-CoV-2 presenten ansiedad por desconocer cuál será la respuesta del organismo ante la enfermedad y que probablemente algunos de los resultados de xerostomía podrían estar condicionados por esta variante ${ }^{13}$. Un $27 \%$ de nuestra muestra refirió este síntoma, en la literatura se ha reportado la presencia de hiposalivación y xerostomía en infectados con SARS$\mathrm{CoV}-2^{14}$.

Este dato es de importancia, ya que, pacientes con hiposalivación poseen un mayor riesgo de desarrollar una infección respiratoria, dado que al existir una menor secreción salival es posible que se altere la función de barrera de la mucosa de la vía aérea respiratoria, favoreciendo la adhesión y colonización viral. A su vez, la hiposalivación se relaciona con una disminución en proteínas salivales, algunas de las cuales pueden potencialmente impedir la replicación del virus, la actividad antiviral en saliva puede ser resultado de micro vesículas de saliva, incluyendo al menos 20 microARN, que pueden restringir la replicación de algunos tipos de virus ${ }^{15}$.

De acuerdo con varias publicaciones científicas el virus SARS-CoV-2 puede causar sialoadenitis aguda de acuerdo con el siguiente proceso. El virus se une a los receptores ECA2 en el epitelio de las glándulas salivales, se fusiona con ellos, se replica y lisa las células para inducir síntomas y signos, como malestar, edema y dolor en las glándulas salivales principales (glándulas parótidas y submandibulares) ${ }^{16,17}$. En nuestro estudio el $17 \%$ de los pacientes presentaban un reporte subjetivo de inflamación de ganglios y/o glándulas salivales.
La frecuencia de lesiones orales no fue estadísticamente significativa, los casos únicamente reportaban la presencia de una úlcera y al no realizar la valoración clínica, desconocemos si pudo asociarse a traumatismos, o a otros virus asociados como el herpes o fue directamente relacionado con el SARS-CoV-2. Aún a pesar de las limitaciones del estudio por no realizar una valoración clínica, La detección de disgeusia y anosmia debe considerarse como un potencial indicador de COVID-19, Esto es de vital importancia a nivel odontológico ya que los entornos de atención dental conllevan riesgos elevados de infección por SARS COV-2 debido a la especificidad de los procedimientos, lo que implica contacto cercano con los pacientes asociado a la exposición frecuente a aerosoles, saliva y otros fluidos tanto del tracto respiratorio como digestivo. Hay evidencia de que las manifestaciones orales, son comunes en personas infectadas con COVID-19; aunque estas se reducen significativamente posterior a su recuperación, algunas persisten. Se sugiere continuar con estudios de seguimiento que nos permitan conocer más a fondo esta interrogante e incluir a personal de odontología que valore clínicamente con mayor precisión estas manifestaciones. La disgeusia es la manifestación oral más prevalente en este estudio y se presentó entre las cinco manifestaciones generales más comunes.

\section{Referencias bibliográficas}

1. Amorim dos Santos J, Normando AGC, Carvalho da Silva RL, Acevedo A, De Luca G, Sugaya N. Oral Manifestations in Patients with COVID-19: A Living Systematic Review. J Dent Res. 2020;100(2):1-14. DOI: $10.1177 / 002203452095728$.

2. Amorim dos Santos J, Normando AGC, Carvalho da Silva RL, De Paula RM, Cembranel AC, Santos-Silva AR, et al. Oral mucosal lesions in a COVID-19 patient: New signs or secondary manifestations? Int J Infect Dis. 2020;97:326-8. DOI: 10.1016/J.IJID.2020.06.012.

3. Zou X. The single-cell RNA-seg data analysis on the receptor ACE2 expression reveals the potential risk of different human organs vulnerable to Wuhan 2019- 
nCoV infection. Front Med. 2020;14(2):185-192. DOI: 10.1007/S11684-020-0754-0.

4. Ou X, Liu Y, Lei X, Li P, Mi D, Guo L, et al. Characterization of spike glycoprotein of SARS-CoV-2 on virus entry and its immune cross-reactivity with SARS-CoV. Nat Commun. 2020;11(1):1620. DOI: 10.1038/s41467020-15562-9.

5. Soto Pastrian G. Presencia y Expresión del Receptor ACE2 (Target de SARS-CoV-2) en Tejidos Humanos y Cavidad Oral . Posibles Rutas de Infección en Órganos Orales. Int J odontostomatol. 2020;14(4):501-7.

6. Mao L, Jin H, Wang M, Hu Y, Chen S, He Q, et al. Neurologic manifestations of hospitalized patients with coronavirus disease 2019 in Wuhan, China. JAMA Neurol [Internet]. 2020;77(6):683-90.

7. Xu H, Zhong L, Deng J, Peng J, Dan H, Zeng X, et al. High expression of ACE2 receptor of 2019-nCoV on the epithelial cells of oral mucosa. Int J Oral Sci. 2020;12(1):8. DOI: 10.1038/S41368-020-0074-X.

8. Lechien JR, Chiesa-Estomba CM, De Siati DR, Horoi $\mathrm{M}$, Le Bon SD, Rodriguez A, et al. Olfactory and gustatory dysfunctions as a clinical presentation of mild-to-moderate forms of the coronavirus disease (COVID-19): a multicenter European study. Eur Arch Oto-Rhino-Laryngology. 2020;277(8):2251-61. DOI: 10.1007/S00405-020-05965-1.

9. Brandao B, Alcino L, Silva T, Prado A, Froelich A. Oral lesions in patients with SARS-CoV-2 infection: could the oral cavity be a target organ?. Oral Surg Oral Med Oral Pathol Oral Radiol. 2021;131(2):e45-e51. DOI: 10.1016/J.OOOO.2020.07.014.

10. Soares CD, Mosqueda-Taylor A, de Carvalho MGF, de Almeida OP. Oral vesiculobullous lesions as an early sign of COVID-19: immunohistochemical detection of SARS-CoV-2 spike protein. Br J Dermatol. 2021;184(1):e6. DOI: 10.1111/BJD.19569.
11. Dell'Era V, Farri F, Garzaro G, Gatto M, Valletti P, Garzaro M. Smell and taste disorders during COVID-19 outbreak: Cross-sectional study on 355 patients. Head Neck. 2020;42(7):1591-6. DOI: 10.1002/HED.26288.

12. Liguori C, Pierantozzi M, Spanetta M, Sarmati L, Cesta $\mathrm{N}$, Iannetta $\mathrm{M}$, et al. Subjective neurological symptoms frequently occur in patients with SARS-CoV2 infection. Brain Behav Immun. 2020;88:11-16. DOI: 10.1016/J. BBI.2020.05.037.

13. Marcial Guiñez Coelho. Impacto del COVID-19 (SARS-CoV-2) a Nivel Mundial, Implicancias y Medidas Preventivas en la Práctica Dental y sus Consecuencias Psicológicas en los Pacientes. Int J Odontostomatol. 2020;14(3):271-277.

14. Nemeth-Kohanszky ME, Matus-Abásolo CP, Carrasco-Soto RR. Manifestaciones Orales de la Infección por COVID-19. Int J Odontostomatol. 2020;14(4):555-60.

15. Baghizadeh M. Oral saliva and COVID-19. Oral Oncol. 2020;108:104821. DOI: 10.1016/j.oraloncology.2020.104821.

16. Wang C, Wu H, Ding X, Ji H, Jiao P, Song H. Does infection of 2019 novel coronavirus cause acute and/or chronic sialadenitis. Med Hypotheses. 2020;(140):109789. DOI: $10.1016 /$ j.mehy.2020.109789.

17. Pedrosa M, Sipert CR, Nogueira FN. Salivary Glands, Saliva and Oral Presentations in COVID-19 infection. Pesqui Bras Odontopediatria Clin Integr. 2020;20(suppl 1):e0104. 